\title{
L'innovation et la course à la nouveauté dans la recherche
}

Cette tribune concerne l'innovation à motivation sociétale ou économique. L'innovation à motivation purement scientifique, telle que celle concernant l'amélioration des détecteurs en physique des particules par exemple, n'est pas concernée par cette analyse.

\section{L'innovation technique et la science}

Il ne fait pas de doute que l'innovation (ici technique) est un atout essentiel d'une industrie compétitive. L'innovation est ce qui permet d'augmenter la performance des dispositifs, de mieux contrôler la fiabilité des procédés, de ménager la matière et l'énergie. L'innovation permet de remplir de nouveaux besoins, voire d'en créer de nouveaux, de capter des marchés, de conquérir des secteurs applicatifs. Tant que nous vivons dans une économie industrielle et libérale, c'est un ingrédient essentiel du succès industriel.

La science est par nature innovante, en ce sens qu'elle porte un regard neuf sur le monde. Sa démarche s'appuie sur la preuve, la vérification et l'évaluation par les pairs. Et s'il est une chose qui est constitutive de notre métier, c'est cette patiente progression, de conjectures en preuve et de preuve en réfutation, qui fait de l'énoncé scientifique une valeur universelle dont la richesse est autant dans la façon de l'élaborer que dans le contenu même.

Dans cette société, la science et la technologie jouent un rôle essentiel, constitutif du progrès technique. Mais nous dénonçons ici une dérive, la contagion du culte de l'innovation depuis le monde industriel, où elle est vitale, vers le monde de la recherche...

\section{La recherche sous l'empire du culte de l'innovation}

Les demandes d'applications sociétales ou de rattachement à des " domaines d'intérêt majeur " sont omniprésentes dans les appels d'offres de la recherche. Et le vocable "innovation " semble envahir toutes les sources de financement, au niveau régional, national, ou européen. Pourquoi donc ce culte de l'innovation, cette obsession de la nouveauté qui semble irriguer toute la politique scientifique actuelle?

Entre tous les sujets potentiellement scientifiquement intéressants, le choix se porte vers ceux que la communauté, scientifique ou plus généralement la société, reconnait comme "valorisants ». Même s'il est bon en recherche d'emprunter des chemins nouveaux, considérer la nouveauté comme une valeur per se a des conséquences sur les modes de fonctionnement de la communauté scientifique - sans toutefois invalider la scientificité des résultats.

Examinons trois effets pervers du culte de la nouveauté et de l'innovation : la " dévalorisation du capital de connaissances acquises ", les "effets de mode et bulles spéculatives scientifiques ", et enfin la «survalorisation de la culture de projet par rapport à la culture de compétences».

\section{Première perversion :}

la dévalorisation du " second capital scientifique "

J'entends par "capital scientifique " l'acquis culturel que donne une formation scientifique et sur lequel se construit une pensée créatrice. Seuls les historiens lisent les documents originaux de Newton ou de Maxwell, mais tous les physiciens ont intégré les résultats de Newton et Maxwell sans passer par les constructions géométriques du premier ou les engrenages fictifs du second. La mécanique classique et l'électromagnétisme font partie du "premier capital scientifique " de tout physicien. Ce "premier capital scientifique " est stable.

Mais le "capital scientifique " se décline en plusieurs niveaux. Dans chaque spécialité existe un corpus de connaissances qui est plus proche, et qui mérite qu'on aille le chercher à sa source. Le culte de la nouveauté ou de l'innovation a des effets immédiats sur la formation des jeunes chercheurs en dévalorisant ce « second capital scientifique " : il n'est pas assez ancien pour avoir été "sacralisé » et ensuite "banalisé », et il est trop vieux pour mériter une attention qu'on ne donne plus qu'à la nouveauté.

Une façon de se convaincre qu'on est innovant est de simplement négliger de connaitre ce qui a déjà été fait. Si la seule chose qui compte est de faire du neuf, et que l'on imagine "faire la course en tête ", il suffit de se tenir au fait de ce qui vient juste d'être publié pour identifier ce qui va bientôt être publié ! Il suffit donc de suivre l'actualité ! Et du coup, on peut en toute bonne conscience « réinventer la roue ".

\section{Deuxième perversion :}

effets de modes et bulles de spéculation intellectuelle Un second aspect, plus grave, du culte de l'innovation, est un biais sur le choix des sujets scientifiques qu'une communauté va mettre en avant. Ce serait une grande naiveté que de penser que le choix d'un domaine de recherche est exclusivement individuel ; ne serait-ce que par la nécessité de disposer des moyens nécessaires à sa recherche, le choix du scientifique s'intègre à une procédure collective. La conséquence des procédures de choix pilotées par le culte de l'innovation est de générer des phénomènes de «bulles spéculatives ». C'est-à-dire des situations dans lesquelles la valeur attribuée par une communauté à un objet n'a qu'un vague rapport avec sa valeur réelle, et que l'attirance pour cet objet résulte essentiellement d'un phénomène d'autoamplification. Le phénomène se produit chaque fois que l'intérêt d'un objet est amplifié par le nombre d'acteurs intéressés. Cet effet de mode, cet engouement collectif n'est pas nécessairement dommageable et irrigue toute l'histoire des sciences (c'est le cas, par exemple, des études sur le graphène et sur les supraconducteurs à haute température critique). 
Mais son amplification hors limite quand les procédures de rétroaction deviennent inopérantes, est relativement récente. La croissance de telles bulles spéculatives est progressive, avec une sortie brutale : il s'agit d'un processus intrinsèquement instable. Les rétroactions possibles résultent des critères secondaires de choix. Entre tous les sujets d'intérêt possibles, ceux qui retiendront préférentiellement l'attention et les activités d'une communauté scientifique structurée seront fortement influencés par ce que la société ou la politique scientifique souhaite valoriser; interviennent alors dans ce choix les critères secondaires que sont la nouveauté intellectuelle, les applications potentielles, la demande sociétale immédiate.

Examinons, pour chacun de ces critères " secondaires ", les mécanismes amplificateurs qui font qu'à un moment donné, une communauté scientifique choisit collectivement de concentrer une part importante de ses efforts à un sujet qui a souvent une " durée de vie " assez réduite, laquelle semble relativement indépendante de la solution ou non du problème. Le culte de l'innovation a pour effet de faire émerger des sujets par le seul fait qu'ils soient nouveaux, et d'en faire disparaitre simplement pour avoir été trop longtemps étudiés. Il justifie ses choix par un raisonnement étrangement circulaire : on lance un programme confortablement doté, qui attire les chercheurs faméliques, et on en déduit que puisqu'il intéresse tant de monde, il était donc juste de doter ainsi ce programme.

Des critères de choix des sujets scientifiques qui ont été déclinés plus haut, seul le premier, "l'attirance pour la nouveauté intellectuelle ", est autorégulé : un sujet nouveau ne le reste pas longtemps, et l'aiguillon de la reconnaissance pour un chercheur s'émousse souvent dès qu'un autre chercheur l'obtient.

Selon le critère "applications potentielles ", la dynamique de recherche peut aussi être régulée dès lors que les acteurs économiques sont effectivement impliqués et que la puissance publique n'endosse pas la totalité des risques. Mais sans cette solide assise économique, le risque existe de comportements spéculatifs sans contre-réaction. Il est en effet facile de s'autoconvaincre du potentiel d'une technologie simplement en lançant de grands programmes dont la seule justification est, in fine, la forte demande de la communauté scientifique pour y participer.

Le critère de la "demande sociétale " est hautement déstabilisant quand il est, comme de plus en plus souvent, la réponse à une crainte de la société ou de tel ou tel groupe de pression. Il est certes du devoir des chercheurs d'éclairer les débats et les controverses. Mais il peut se produire que la multiplicité des études - prenons le cas des OGM - ne conduise qu'à accentuer l'inquiétude : un laboratoire public chargé d'étudier la nocivité potentielle d'un produit ne pourra pas garantir son innocuité totale, un doute subsistera.

L'apparition des "bulles spéculatives ", plus ou moins violentes suivant la maigreur des subsides récurrents (qui induit le terme déstabilisant) et l'intensité du terme de rétroaction (minimal dans le cas des exigences sociétales), a des conséquences importantes sur le bon usage des deniers publics. Mais il y a une conséquence plus grave du culte de l'innovation... Plus grave, car elle porte sur le mode de travail même de la communauté scientifique.

\section{La perversion ultime :}

\section{la déstructuration du socle de compétences}

Dans une logique de pilotage par "projets innovants ", les thématiques «porteuses " mobilisent des compétences, mais n'aident pas nécessairement à les ressourcer. La créativité ne se nourrit pas d'une succession de "success stories" d'innovation, elle a besoin du terreau scientifique issu de la dynamique propre de la science, la première de nos trois motivations. Le risque de voir disparaitre le socle scientifique sans lequel il est simplement impossible de répondre à la société ou au marché n'est pas négligeable.

L'économie de la connaissance qui est devenue un lieu commun à tous les politiques a pour effet de voir transférer d'un côté à l'autre de l'Atlantique, de Washington à Bruxelles, les mêmes mots clés, les mêmes thématiques prioritaires. La création de revues de plus en plus spécialisées, la pression sur les revues généralistes de chercher le sujet "sexy" qui fera remonter son indice de citation est à terme une incitation à la fragmentation des disciplines, alors que l'idée nouvelle résulte souvent de la comparaison. L'obligation de choisir ses sujets non plus dans une dynamique propre à la science ou à ses domaines applicatifs, mais dans une relecture de ses domaines via un prisme politique ou économique a un effet très tendancieux : le scientifique par la force des choses, ne choisira plus ses sujets que par procuration. Il devient virtuose dans l'art de repeindre ses projets de la couleur appropriée, amplifiant de facto les effets de mode.

\section{Conclusion}

Les conséquences majeures des "bulles spéculatives " résultant du culte de l'innovation sont de déstructurer les disciplines scientifiques, de mobiliser toutes les forces sur un sujet identifié comme prioritaire. Il est impératif de sortir d'une analyse de pertinence des sujets uniquement en termes d'objectifs, pour réintroduire la notion de compétences. Faute de quoi les compétences s'étioleront et l'on ne pourra plus répondre aux objectifs que par un psiccatisme de mots clés.

C'est en développant un corpus de compétences, disciplinaires ou interdisciplinaires, que la communauté scientifique est en état de répondre aux demandes de la société et de l'économie. C'est en insistant à nouveau sur cet aspect, et en y consacrant une part importante des financements, que l'on pourra éviter les conséquences les plus délétères des effets de mode.

Cibler la structure de financement de la recherche en suivant uniquement des grilles de lecture "projets innovants » aurait pour effet à moyen et long terme d'éteindre ce qui rend l'innovation réelle possible : un socle scientifique disciplinaire solide et construit dans la durée.

Yves Bréchet

Membre de l'Académie des sciences 